\title{
Quantitative Analysis of Distribution of the Gastrointestinal Tract Eosinophils in Childhood Functional Abdominal Pain Disorders
}

\author{
Eun Hye Lee, ${ }^{1,2}$ Hye Ran Yang, ${ }^{3,4 *}$ and Hye Seung Lee ${ }^{5,6}$ \\ ${ }^{1}$ Department of Pediatrics, Nowon Eulji Medical Center, Seoul, Korea; ${ }^{2}$ Department of Pediatrics, Eulji University College of Medicine, Daejeon, \\ Korea; ${ }^{3}$ Department of Pediatrics, Seoul National University Bundang Hospital, Seongnam, Gyeonggi-do, Korea; ${ }^{4}$ Department of Pediatrics, Seoul \\ National University College of Medicine, Seoul, Korea; ${ }^{5}$ Department of Pathology, Seoul National University Bundang Hospital, Seongnam, \\ Gyeonggi-do, Korea; and ${ }^{6}$ Department of Pathology, Seoul National University College of Medicine, Seoul, Korea
}

\section{Background/Aims}

Although functional abdominal pain disorders (FAPDs) are common in children, the accurate pathogenesis of FAPDs is not known yet. Micro-inflammation, particularly tissue eosinophilia of gastrointestinal (GI) tract, has been suggested as the pathophysiology observed in several GI disorders. We aimed to evaluate eosinophilic infiltration throughout the entire GI tract in children with FAPDs, compared to those with inflammatory bowel diseases (IBD) and to normal reference values.

\section{Methods}

We included 56 children with FAPDs, 52 children with Crohn's disease, and 23 children with ulcerative colitis. All subjects underwent esophagogastroduodenoscopic and colonoscopic examination with biopsies. Tissue eosinophil counts were assessed in 10 regions throughout the Gl tract.

\section{Results}

Eosinophil counts of the gastric antrum, duodenum, terminal ileum, cecum, and ascending colon were significantly higher in children with FAPDs compared to normal reference values. Eosinophil counts of the stomach and the entire colon were observed to be significantly higher in children with IBD than in those with FAPDs. Even after selecting macroscopically uninvolved GI segments on endoscopy in children with IBD, eosinophil counts of the gastric body, cecum, descending colon, sigmoid colon, and the rectum were also significantly higher in children with IBD than those with FAPDs.

\section{Conclusions}

Significantly high eosinophil counts of the stomach and colon were observed in the order of IBD, followed by FAPDs, and normal controls, regardless of endoscopically detected macroscopic IBD lesions in children. This suggests some contribution of GI tract eosinophils in the intrinsic pathogenesis of FAPDs in children.

(J Neurogastroenterol Motil 2018;24:614-627)

\section{Key Words}

Abdominal pain; Child; Eosinophils; Functional gastrointestinal disorder; Inflammatory bowel diseases

Received: March 3, 2018 Revised: July 13, 2018 Accepted: July 31, 2018

@) This is an Open Access article distributed under the terms of the Creative Commons Attribution Non-Commercial License (http://creativecommons. org/licenses/by-nc/4.0) which permits unrestricted non-commercial use, distribution, and reproduction in any medium, provided the original work is properly cited.

*Correspondence: Hye Ran Yang, MD, PhD Department of Pediatrics, Seoul National University Bundang Hospital, Seoul National University, 82, Gumi-ro 173 Beon-gil, Bundang-gu, Seongnam, Gyeonggi-do 13620, Korea Tel: +82-31-787-7285, Fax: +82-31-787-4054, E-mail: hryang@snubh.org 


\section{Introduction}

Functional abdominal pain disorders (FAPDs) are common in children. A recent meta-analysis has reported a global pooled prevalence rate for FAPDs was $16.5 \%$ based on the Rome III criteria. ${ }^{1}$ It is well-known that FAPDs could be associated with long-term symptoms. A prospective study has demonstrated that approximately $41.0 \%$ of children with functional abdominal pain (FAP) continued to report clinically significant abdominal pain after a mean duration of a 9-year follow-up into adolescence and young adulthood. ${ }^{2,3}$

The pathomechanism to explain FAPDs remains unclear and multifactorial hypotheses have been suggested. ${ }^{4,5}$ Recently many studies have suggested that low-grade intestinal inflammation is essentially involved in the underlying pathogenesis of functional gastrointestinal disorders (FGIDs). ${ }^{6,7}$ These studies have reported that increased infiltration of immune cells such as eosinophils, mast cells, and lymphocytes and the subsequent degranulation of these activated immune cells produce low-grade mucosal inflammation in the gastrointestinal (GI) tract of adults with functional dyspepsia (FD) and/or irritable bowel syndrome (IBS). However, the types of immune cells that were increased, the degree of inflammation, and the segments of the GI tract that were investigated have not been consistent across all these studies performed in adults. Furthermore, histopathological studies pertaining to childhood FAPDs are relatively few, and studies involving children are primarily limited to studies regarding FD. ${ }^{8,9}$

In our previous study published in 2016, we demonstrated that eosinophil counts in the gastric antrum and body mucosa were significantly high in a definite order of in terms of the disease entity normal reference values, followed by children with FAPDs, and then those with Helicobacter pylori gastritis. ${ }^{10}$ In the present study, we extended the scope of our study to investigate the entire GI tract. Unlike the esophagus, eosinophils are resident cells of the stomach, small and large intestine. However, to date, the normal range of eosinophils in the GI tract mucosa is not well-defined; thus, interpretation of pathological intestinal eosinophilia is difficult. ${ }^{11}$ It is also known that eosinophils are not uniformly distributed throughout the length of the intestine. ${ }^{12}$ Therefore, eosinophils of the GI tract should be analyzed and compared in each specific region, even within the colon.

We aim to evaluate tissue eosinophilic infiltration of the entire GI tract from the stomach through the rectum in children with FAPDs, compared to the normal reference values and disease controls with inflammatory bowel diseases (IBD) in each segment throughout the bowel.

\section{Materials and Methods}

\section{Subjects}

Our retrospective study included children and adolescents who visited the Department of Pediatric Gastroenterology at the Seoul National University Bundang Hospital with complaints of chronic recurrent abdominal pain, and who had undergone both esophagogastroduodenoscopic and colonoscopic examination with biopsies due to accompanied red flag signs, and finally had been diagnosed with FAPDs between January 2009 and October 2015. Children excluded from this study were those with a significant allergy such as allergic rhinitis, atopic dermatitis, asthma, and food allergy that may affect tissue eosinophils in the GI tract, and those with underlying liver, kidney or heart disease that could cause GI symptoms.

All children underwent laboratory tests including complete blood cell counts, erythrocyte sedimentation rate, C-reactive protein, liver function tests, serum amylase and lipase, serum electrolytes, urinalysis, stool examination for parasite, fecal occult blood, and stool cultures for bacteria, as well as abdominal X-ray and ultrasonography. Those showing abnormalities on laboratory tests and imaging studies were excluded. Children who demonstrated endoscopically any macroscopic abnormality were also excluded from the study. Eventually, 56 children with FAPDs were included and classified into 4 subtypes of FAPDs including FD, IBS, abdominal migraine (AM), and FAP-not otherwise specified (FAP-NOS) based on the Rome IV criteria. ${ }^{4}$

Among the children who had been diagnosed with IBD and had been treated in our tertiary medical center, those who underwent both esophagogastroduodenoscopic and colonoscopic examination with biopsies during the same period were also recruited retrospectively as disease controls. Eventually, 52 children with Crohn's disease (CD) and 23 children with ulcerative colitis (UC) were enrolled. We used the study reported by DeBrosse et $\mathrm{al}^{12}$ as an upper GI reference to compare eosinophil infiltration between the stomach and ileum and another study reported by $\mathrm{Saad}^{13}$ as a lower GI reference to compare colonic eosinophil counts.

Our retrospective study was approved by the Institutional Review Board of Seoul National University Bundang Hospital (IRB No. B-1609-363-102). 


\section{Endoscopic Biopsy and Histopathology}

Esophagogastroduodenoscopy was performed using a GIFQ260 or GIF-XP260 scope (Olympus, Tokyo, Japan) and colonoscopy was performed using a PCF-Q260AL, GIF-Q260, or GIF-XP260 scope (Olympus). Endoscopic mucosal biopsies were obtained from the esophagus, gastric antrum and body, duodenum, terminal ileum, cecum, ascending, transverse, descending, and sigmoid colon, and the rectum.

Biopsy tissues were immediately fixed using formalin and processed with embedding in paraffin wax. Sections of size $3 \mu \mathrm{m}$ were cut from the paraffin block, and stained using hematoxylin and eosin stains. The eosinophil count was obtained from 5 randomly selected high-power fields. Quantification of eosinophils was performed using an Axioskope40 microscope (Mirax-Carl Zeiss, Oberkochen, Germany) at $\times 400$ magnification. Cell counts were performed by 2 pathologists who were blinded to the status of the children, and a mean value of the 5 high-power fields counts obtained was calculated for each child (Fig. 1).

Because it is known that eosinophils are not usually observed in the esophageal mucosa in a non-pathological state, we excluded the esophagus from the areas that were compared. Mucosal eosinophil counts in each of the 10 regions of the GI tract of children with FAPDs were compared with those in normal pathology references (normal controls) and those from children with IBD (disease controls), respectively.

To minimize the confounding effects of IBD-induced inflammation, after selecting histopathological specimens of the GI tract segments that endoscopically did not show macroscopic IBD involvement, we analyzed eosinophil counts as well. Macroscopically uninvolved segments of GI tract were selected based on use of 4 variables of the simple endoscopic score for $\mathrm{CD}(\mathrm{SES}-\mathrm{CD})^{14}$ and 3 descriptors of UC endoscopic index of severity (UCEIS). ${ }^{15,16}$ The segments in which all scores of all 4 variables pertaining to SES$\mathrm{CD}$ and of all 3 descriptors pertaining to UCEIS were 0 were selected for analysis after eliminating IBD-induced regional inflammation.

\section{Statistical Methods}

Data for continuous variables are presented as mean $\pm \mathrm{SD}$ for parametric or median values along with range (minimummaximum) for nonparametric variables. Categorical variables are presented as a percentage of the total number. Continuous data were analyzed using the independent $t$ test for parametric variables, and the Mann-Whitney $U$ test for nonparametric variables. For all statistical analyses, a two-sided $P$-value $<0.05$ was considered statistically significant. All statistical analyses were performed using PASW Statistics (version 20.0; IBM Corp, Armonk, NY, USA).
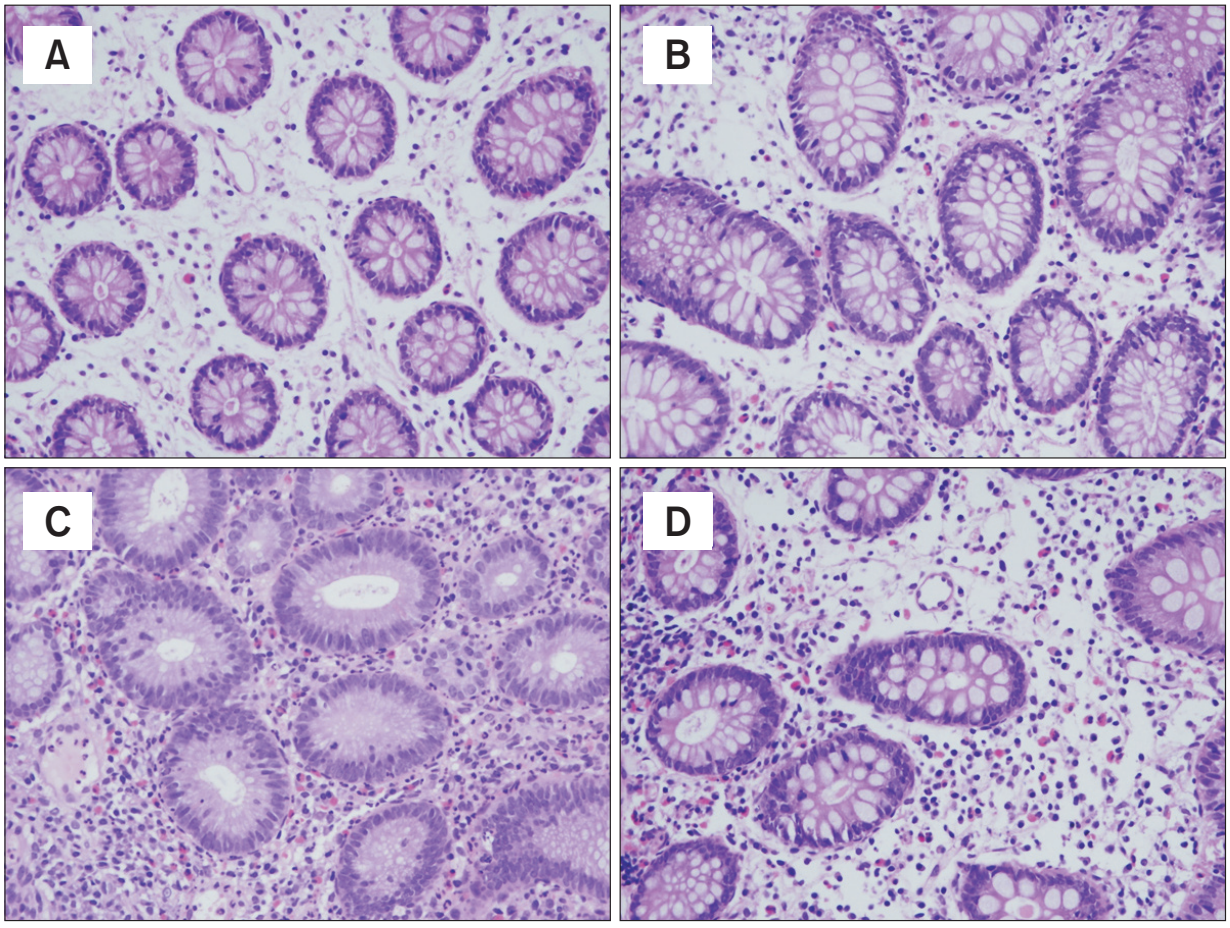

Figure 1. Photomicrograph of hematoxylin and eosin staining for eosinophils in the ascending colon. Infiltration of tissue eosinophils increased in a definite order of specimen of normal controls (A), functional abdominal pain disorders (B), Crohn's disease (C), and ulcerative colitis (D) (magnification, $\times 400)$. 


\section{Results}

\section{Patients Characteristics}

Table 1 shows the demographic data of the subjects. Our study included 56 children fulfilling the Rome IV criteria for FAPDs who were classified as IBS $(\mathrm{n}=37), \operatorname{IBS}+\mathrm{FD}(\mathrm{n}=5)$, IBS + FAP-NOS $(\mathrm{n}=3), \operatorname{IBS}+\operatorname{AM}(\mathrm{n}=1)$, FAP-NOS $(\mathrm{n}=6)$, FD $(n=3)$, and AM $(n=1)$. Normal controls for the upper GI tract were 19 children and those for the lower GI tract were 41 children. Disease controls with IBD comprised 52 children with CD and 23 children with UC.

\section{Comparison of Eosinophil Counts in the Gastrointestinal Tract Between Patients With Functional Abdominal Pain Disorders and Those With Inflammatory Bowel Diseases}

Eosinophil counts of the stomach and the entire colon between cecum and the rectum were significantly higher in children with IBD than in those with FAPDs (Table 2 and Fig. 2). Eosinophil counts of the duodenum and the terminal ileum were also higher in children with IBD, but this was not a significant difference (Table 2 and Fig. 2).

Comparisons between children with FAPDs and those with $\mathrm{CD}$ as well as those with UC are also shown in Table 2. A comparison between children diagnosed with FAPDs and those with CD showed that the eosinophil counts of the stomach (both gastric antrum and body) and the left colon (transverse colon, descending colon, sigmoid colon, and rectum) in children with $\mathrm{CD}$ were

Table 1. Demographic Characteristics of the Subjects Recruited

\begin{tabular}{lcccc}
\hline \multicolumn{1}{c}{ Variable } & $\begin{array}{c}\text { FAPDs } \\
(\mathrm{n}=56)\end{array}$ & $\begin{array}{c}\text { Normal reference } \\
(\text { upper GI, } \mathrm{n}=19)\end{array}$ & $\begin{array}{c}\text { Normal reference } \\
(\text { lower GI, } \mathrm{n}=41)\end{array}$ & $\begin{array}{c}\text { IBD }(\mathrm{CD}+\mathrm{UC}) \\
(\mathrm{n}=75)\end{array}$ \\
\hline Sex (boys:girls) & $39: 17$ & $8: 11$ & $21: 20$ & $55: 20$ \\
Age (median [range], yr) & $14.1(2.8-18.9)$ & $12.0(2.0-17.0)$ & $12.2(3.3-17.9)$ & $14.7(2.5-19.5)$ \\
\hline
\end{tabular}

FAPDs, functional abdominal pain disorders; upper GI, upper gastrointestinal region including gastric antrum, gastric body, duodenum, and terminal ileum; lower GI, lower gastrointestinal region including cecum, ascending colon, transverse colon, descending colon, and rectosigmoid colon; IBD, inflammatory bowel disease; $\mathrm{CD}$, Crohn's disease; UC, ulcerative colitis.

Age is shown as median value along with range (minimum-maximum).

Table 2. Comparison of Tissue Eosinophil Counts of the Gastrointestinal Tract Between Children With Functional Abdominal Pain Disorders and Those With Inflammatory Bowel Diseases

\begin{tabular}{|c|c|c|c|c|c|c|c|}
\hline Anatomical region & $\begin{array}{l}\text { FAPDs } \\
(\mathrm{n}=56)\end{array}$ & $\begin{array}{c}\mathrm{CD} \\
(\mathrm{n}=52)\end{array}$ & $\begin{array}{c}\text { UC } \\
(n=23)\end{array}$ & $\begin{array}{c}\mathrm{IBD}(\mathrm{CD}+\mathrm{UC}) \\
(\mathrm{n}=75)\end{array}$ & $\begin{array}{c}{ }^{\mathrm{a}} \mathrm{P} \text {-value } \\
(\mathrm{FAPDs}-\mathrm{CD})\end{array}$ & $\begin{array}{c}{ }^{\mathrm{b}} P \text {-value } \\
\text { (FAPDs-UC) }\end{array}$ & $\begin{array}{c}{ }^{\mathrm{c} P} \text {-value } \\
\text { (FAPDs-IBD) }\end{array}$ \\
\hline Gastric antrum & $4.1 \pm 6.1$ & $11.2 \pm 13.0$ & $5.4 \pm 5.1$ & $9.5 \pm 11.5$ & 0.001 & 0.207 & 0.001 \\
\hline Gastric body & $2.6 \pm 2.5$ & $7.0 \pm 9.7$ & $5.0 \pm 6.6$ & $6.4 \pm 8.9$ & 0.004 & 0.104 & 0.001 \\
\hline Duodenum & $13.0 \pm 8.5$ & $18.5 \pm 19.4$ & $15.0 \pm 9.3$ & $17.4 \pm 17.1$ & 0.078 & 0.389 & 0.068 \\
\hline Terminal ileum & $22.3 \pm 17.6$ & $27.8 \pm 17.7$ & $19.1 \pm 9.6$ & $25.5 \pm 16.3$ & 0.291 & 0.983 & 0.488 \\
\hline Cecum & $23.0 \pm 19.2$ & $27.0 \pm 17.3$ & $34.3 \pm 18.6$ & $29.4 \pm 17.9$ & 0.382 & 0.033 & $0.047^{\mathrm{d}}$ \\
\hline Ascending colon & $15.7 \pm 9.5$ & $20.4 \pm 17.8$ & $27.3 \pm 15.9$ & $22.7 \pm 17.4$ & 0.140 & 0.002 & 0.011 \\
\hline Transverse colon & $13.1 \pm 9.1$ & $23.4 \pm 20.8$ & $27.3 \pm 20.1$ & $24.6 \pm 20.5$ & 0.004 & 0.005 & $<0.001$ \\
\hline Descending colon & $12.7 \pm 8.8$ & $19.5 \pm 15.3$ & $31.8 \pm 17.8$ & $23.4 \pm 17.0$ & 0.014 & $<0.001$ & $<0.001$ \\
\hline Sigmoid colon & $11.9 \pm 12.8$ & $18.8 \pm 16.7$ & $29.3 \pm 20.9$ & $22.0 \pm 18.6$ & 0.021 & $<0.001$ & 0.001 \\
\hline Rectum & $3.3 \pm 3.0$ & $7.8 \pm 9.8$ & $25.9 \pm 26.3$ & $13.1 \pm 18.1$ & 0.003 & $<0.001$ & $<0.001$ \\
\hline
\end{tabular}

${ }^{a} P$-value between functional abdominal pain disorders (FAPDs) and Crohn's disease (CD) was calculated by independent $t$ test.

${ }^{\mathrm{b}} P$-value between FAPDs and ulcerative colitis (UC) was calculated by independent $t$ test.

${ }^{\mathrm{c}} P$-value between FAPDs and inflammatory bowel disease (IBD) (CD $\left.+\mathrm{UC}\right)$ was calculated by independent $t$ test except for ${ }^{\mathrm{d}} P$-value by Mann-Whitney $U$ test.

Data indicate the mean number of eosinophils/high-power field for each anatomical region of the gastrointestinal tract. Data are shown as mean \pm SD. 


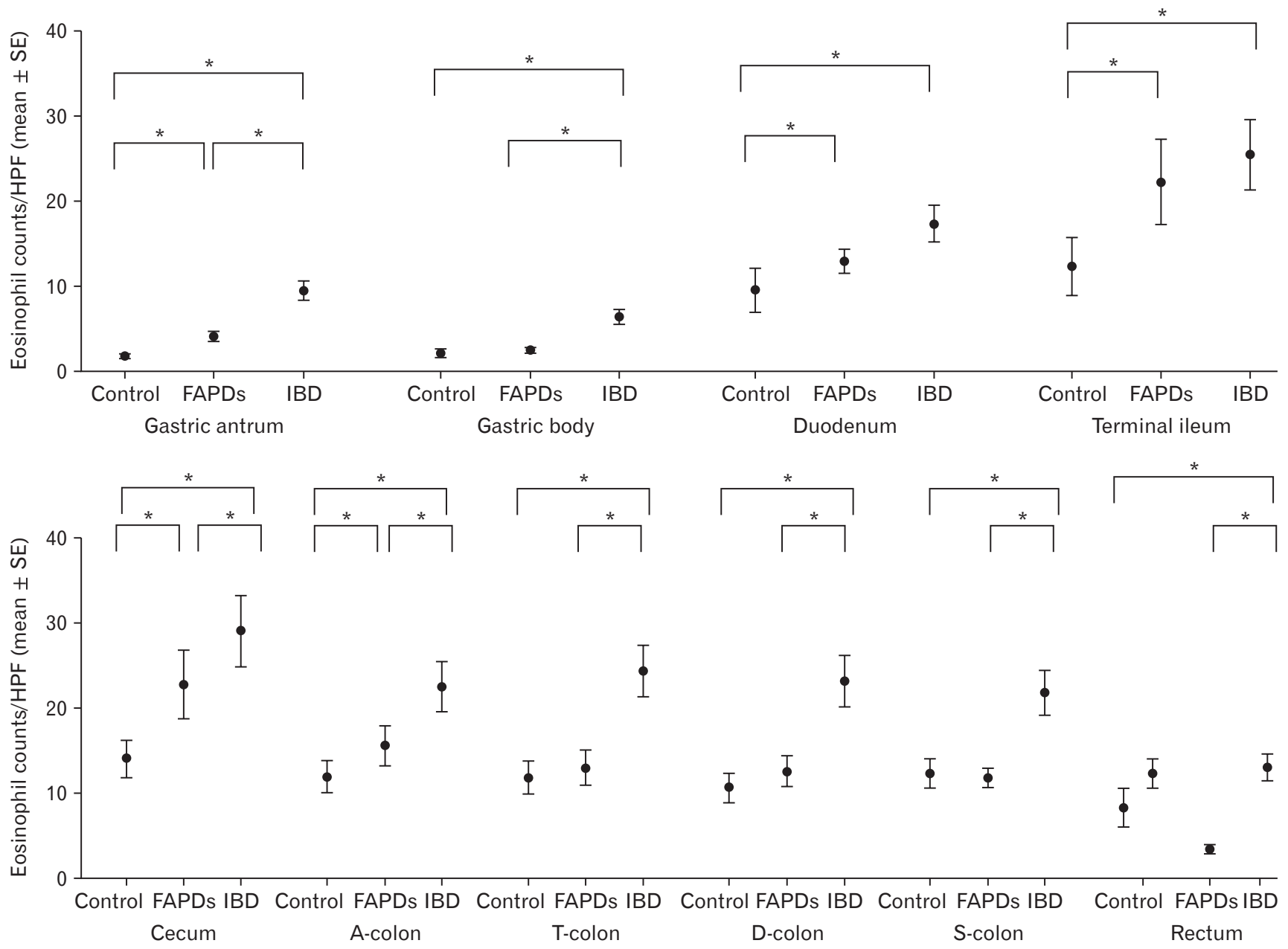

Figure 2. Eosinophil counts in each part of the gastrointestinal tract in normal controls, children with functional abdominal pain disorders (FAPDs), and children with inflammatory bowel diseases (IBD). The order of bar graphs is normal controls, FAPDs, and IBD. In the rectum, eosinophil counts according to both upper gastrointestinal (GI) reference and lower GI reference were presented because lower GI tract pathology reference presented the "rectosigmoid area," with no clear division between the sigmoid and the rectum. * $P$-value is less than 0.05 . HPF, high-power field; A-colon, ascending colon; T-colon, transverse colon; D-colon, descending colon; S-colon, sigmoid colon.

significantly higher than those observed in children with FAPDs (Table 2). Eosinophil counts of the duodenum, terminal ileum, cecum, and the ascending colon were also higher in children with $\mathrm{CD}$ than those observed in children with FAPDs, although this difference was not statistically significant. A comparison between children diagnosed with FAPDs and those with UC showed that eosinophils throughout the colon between the cecum and rectum were significantly higher in children with UC than in children with FAPDs (Table 2). Eosinophil counts of the stomach and small bowel (duodenum and terminal ileum) were not significantly different between children with FAPDs and those with UC (Table 2).

To minimize the confounding effects of IBD-induced inflammation, after histopathological slides of macroscopically uninvolved
GI segments on endoscopy were selected, eosinophil counts were analyzed between children with FAPDs and those with IBD as well. Eosinophil counts in children with IBD were significantly higher than those observed in children with FAPDs in the gastric body, cecum, descending colon, sigmoid colon, and rectum (Table 3 and Fig. 3). Eosinophil counts of the ascending colon and transverse colon were also higher in children with IBD, but this difference was not statistically significant. Eosinophil counts of the small bowel (duodenum and terminal ileum) were not significantly different between children with FAPDs and those with IBD. Comparisons between children with FAPDs and those with $\mathrm{CD}$ as well as those with UC after eliminating the specimens of the GI segments with IBD lesions on endoscopy are shown in Table 3. A comparison 
Table 3. Comparison of Gastrointestinal Tract Mucosal Eosinophil Counts Between Children With Functional Abdominal Pain Disorders and Inflammatory Bowel Diseases Except Regional Inflammation Site

\begin{tabular}{|c|c|c|c|c|c|c|c|}
\hline Anatomical region & $\begin{array}{l}\text { FAPDs } \\
(\mathrm{n}=56)\end{array}$ & $\begin{array}{l}\text { CD except regional } \\
\text { inflammation } \\
(\mathrm{n}=52)\end{array}$ & $\begin{array}{l}\text { UC except regional } \\
\text { inflammation } \\
(\mathrm{n}=23)\end{array}$ & $\begin{array}{l}\text { IBD except regional } \\
\text { inflammation } \\
(\mathrm{n}=75)\end{array}$ & $\begin{array}{l}{ }^{\mathrm{a}} P \text {-value } \\
\text { (FAPDs- } \\
\text { CD) }\end{array}$ & $\begin{array}{l}{ }^{\mathrm{b}} \mathrm{P} \text {-value } \\
\text { (FAPDs- } \\
\text { UC) }\end{array}$ & $\begin{array}{l}{ }^{\mathrm{c}} P \text {-value } \\
\text { (FAPDs- } \\
\text { IBD) }\end{array}$ \\
\hline Gastric antrum & $4.1 \pm 6.1$ & $5.8 \pm 4.1$ & $5.6 \pm 5.1$ & $5.8 \pm 4.4$ & $0.002^{\mathrm{d}}$ & 0.141 & 0.096 \\
\hline Gastric body & $2.6 \pm 2.5$ & $5.3 \pm 5.7$ & $5.2 \pm 6.8$ & $5.3 \pm 6.0$ & $0.007^{\mathrm{d}}$ & 0.088 & 0.002 \\
\hline Duodenum & $13.0 \pm 8.5$ & $11.4 \pm 6.9$ & $13.7 \pm 8.6$ & $12.2 \pm 7.5$ & 0.365 & 0.686 & 0.614 \\
\hline Terminal ileum & $22.3 \pm 17.6$ & $25.5 \pm 10.1$ & $17.6 \pm 8.7$ & $22.2 \pm 10.1$ & 0.562 & 0.813 & $0.496^{\mathrm{d}}$ \\
\hline Cecum & $23.0 \pm 19.2$ & $29.9 \pm 18.7$ & $35.9 \pm 16.7$ & $31.6 \pm 18.0$ & 0.228 & 0.050 & $0.032^{\mathrm{d}}$ \\
\hline Ascending colon & $15.7 \pm 9.5$ & $17.1 \pm 12.6$ & $28.1 \pm 16.4$ & $20.1 \pm 14.3$ & 0.597 & 0.020 & 0.117 \\
\hline Transverse colon & $13.1 \pm 9.1$ & $18.3 \pm 11.4$ & $13.6 \pm 7.5$ & $17.2 \pm 10.7$ & 0.044 & 0.671 & 0.076 \\
\hline Descending colon & $12.7 \pm 8.8$ & $18.8 \pm 18.1$ & $21.1 \pm 10.7$ & $19.4 \pm 16.6$ & 0.053 & 0.021 & 0.021 \\
\hline Sigmoid colon & $11.9 \pm 12.8$ & $15.7 \pm 10.6$ & $23.4 \pm 16.4$ & $17.4 \pm 12.3$ & 0.178 & 0.020 & 0.046 \\
\hline Rectum & $3.3 \pm 3.0$ & $5.8 \pm 4.4$ & $29.0 \pm 37.9$ & $9.2 \pm 16.3$ & 0.002 & 0.021 & 0.027 \\
\hline
\end{tabular}

${ }^{a} P$-value between functional abdominal pain disorders (FAPDs) and Crohn's disease (CD) except regional inflammatory sites was calculated by independent $t$ test except for ${ }^{\mathrm{d}} P$-value by Mann-Whitney $U$ test.

${ }^{b} P$-value between FAPDs and ulcerative colitis (UC) except regional inflammatory sites was analyzed by Mann-Whitney $U$ test.

${ }^{c} P$-value between FAPDs and inflammatory bowel disease (IBD) except regional inflammatory sites was calculated by independent $t$ test except for ${ }^{\mathrm{d}} P$-value by Mann-Whitney $U$ test.

Data indicate the mean number of eosinophils/high-power field for each anatomical region of the gastrointestinal tract. Data are shown as mean \pm SD.

between children with FAPDs and those with CD after eliminating the specimens of the GI segments with macroscopic lesions, showed that the eosinophil counts of the stomach (both gastric antrum and body) and 2 segments of the large intestine (transverse colon and rectum) in children with $\mathrm{CD}$ were significantly higher than those observed in children with FAPDs (Table 3). A comparison between children with FAPDs and those with UC after eliminating the specimens of the GI segments with macroscopic lesions, showed that the eosinophils in sections of the colon (ascending colon, descending colon, sigmoid colon, and rectum) were significantly higher in children with UC than those observed in children with FAPDs (Table 3).

A comparison between children with $\mathrm{CD}$ and those with $\mathrm{UC}$ showed that children with UC demonstrated significantly higher numbers of eosinophils in the ascending colon, descending colon, sigmoid colon, and rectum than those observed in children with CD. However, after excluding the tissue specimens with macroscopically active inflammatory lesions, there were no significant differences in tissue eosinophil counts except the ascending colon (data are not shown).

\section{Comparison of Tissue Eosinophil Counts Among Normal Controls and Those With Functional Abdominal Pain Disorders and Inflammatory Bowel Diseases}

A comparison between children with FAPDs and normal controls showed that eosinophil counts of children with FAPDs were significantly higher in the stomach (gastric antrum), small bowel (duodenum, terminal ileum), cecum, and the ascending colon than those observed in normal controls (Table 4 and Fig. 2).

A comparison between children with IBD and normal controls showed that eosinophil counts in all segments between the stomach and the rectum were significantly higher in children with IBD than those observed in normal controls (Table 4 and Fig. 2). Because the study reported by $\mathrm{Saad}^{13}$ which we used as the lower GI pathology reference presented a 'rectosigmoid area,' and the region was not definitively divided into the sigmoid and rectum, it was difficult to accurately compare the eosinophil counts in the rectum. Eosinophil counts of the rectum were significantly higher in children with IBD than in normal controls when the rectal eosinophils were compared using the values noted by the DeBrosse et $\mathrm{al}^{12}$ (Table 4 and Fig. 2). Comparisons between normal controls and children with UC as well as those with CD are shown in Table 4. A comparison between children with UC and those studied as normal controls showed that the eosinophil counts throughout the GI tract between the stomach and rectum were significantly higher in children with $\mathrm{UC}$ than in 


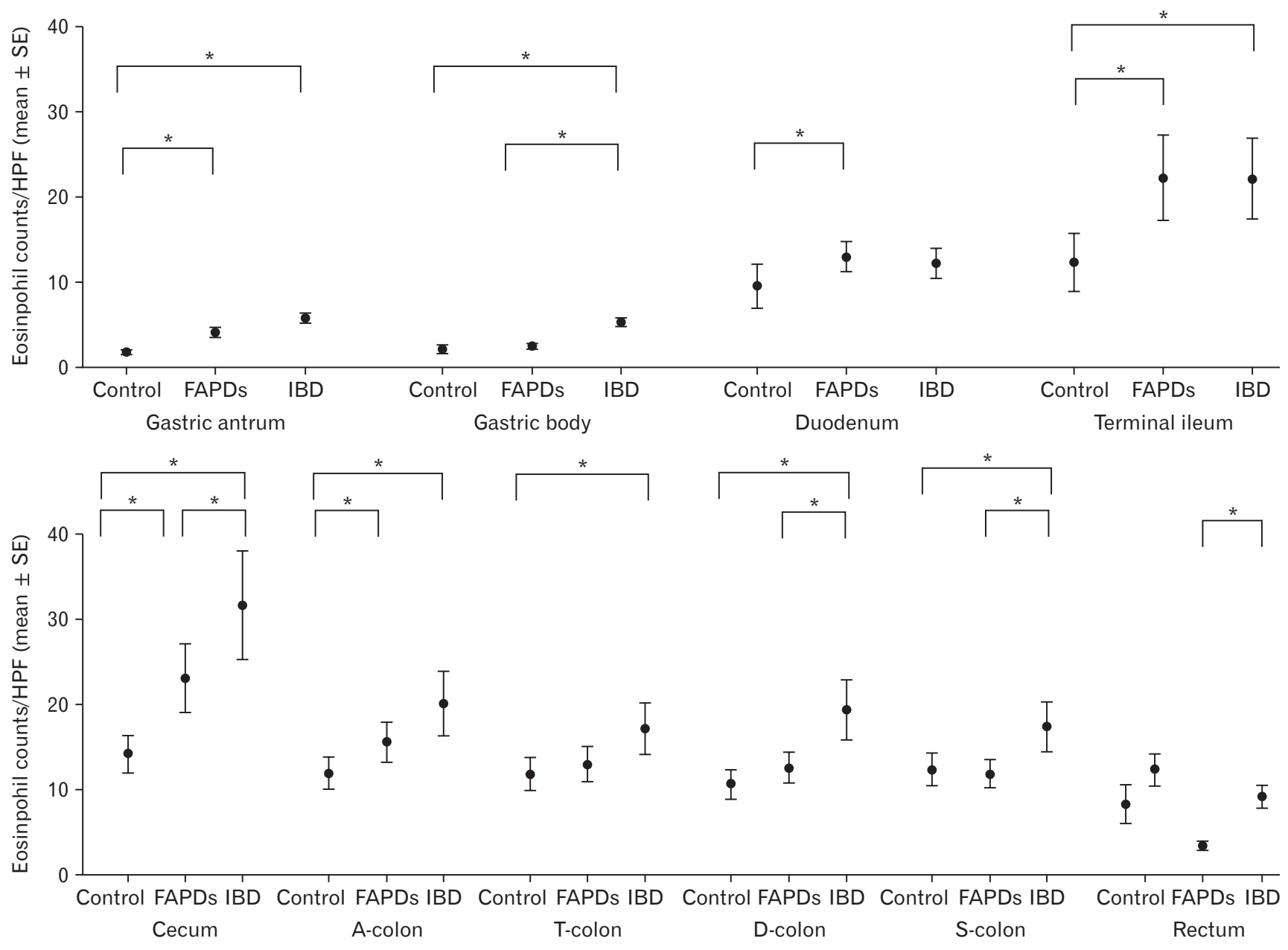

Figure 3. Eosinophil counts in each part of the gastrointestinal (GI) tract in normal controls, childrens with functional abdominal pain disorders (FAPDs), and children with inflammatory bowel diseases (IBD) after eliminating the GI segments with grossly affected IBD lesion on endoscopy. The order of bar graphs is normal controls, FAPDs, and IBD. In the rectum, eosinophil counts according to both upper GI reference and lower GI reference were presented because lower GI tract pathology reference presented the "rectosigmoid area," with no clear division between the sigmoid and the rectum. * $P$-value is less than 0.05. HPF, high-power field; A-colon, ascending colon; T-colon, transverse colon; D-colon, descending colon; S-colon, sigmoid colon.

those studied as normal controls (Table 4). A comparison between children with $\mathrm{CD}$ and those studied as normal controls showed that the eosinophil counts between the stomach and the sigmoid colon were significantly higher in children with $\mathrm{CD}$ than in those studied as normal controls (Table 4). Eosinophil counts of the rectum were not significantly different between children with $\mathrm{CD}$ and those studied as normal controls even when the rectal eosinophils were compared using the values obtained from the pediatric upper GI tract pathology references (Table 4).

After selecting the histopathological specimens of the GI tract segments that endoscopically did not show macroscopic IBD involvement, we analyzed eosinophil counts between normal controls and children with IBD, in these segments to minimize the effects of IBD-induced local inflammation. Eosinophil counts in the GI segments without macroscopic IBD lesions were significantly higher than those of normal controls in the gastric antrum, gastric body, terminal ileum, and throughout the colon except the rectum (Table 5 and Fig. 3). Eosinophil counts in the duodenum were also higher in children with IBD without macroscopic lesions, but not significantly different when compared to normal controls (Table 5 and Fig. 3).

Comparisons between normal controls and children with $\mathrm{CD}$ as well as those with UC after excluding GI segments with macroscopic IBD lesions on endoscopy are also shown in Table 5. A com- 


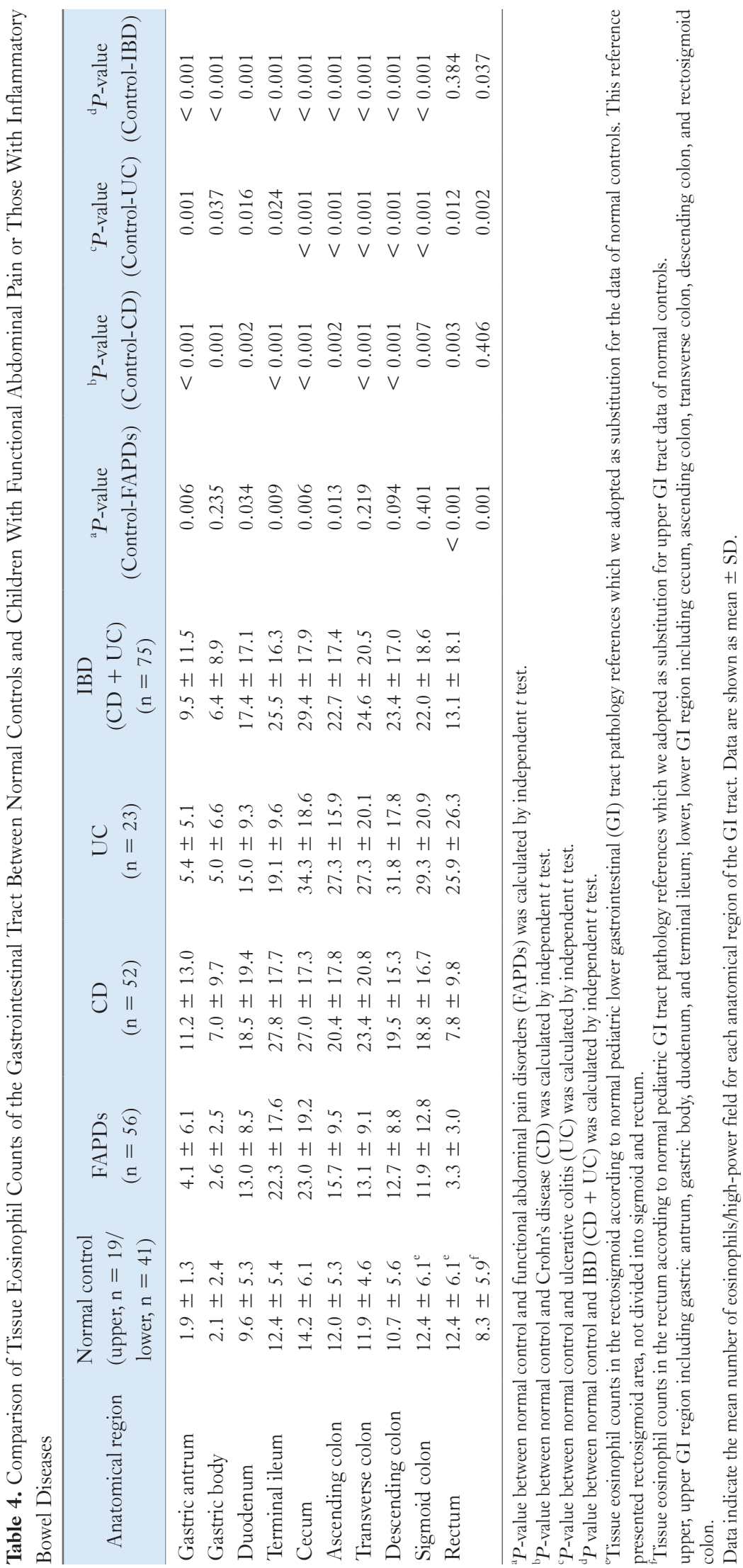




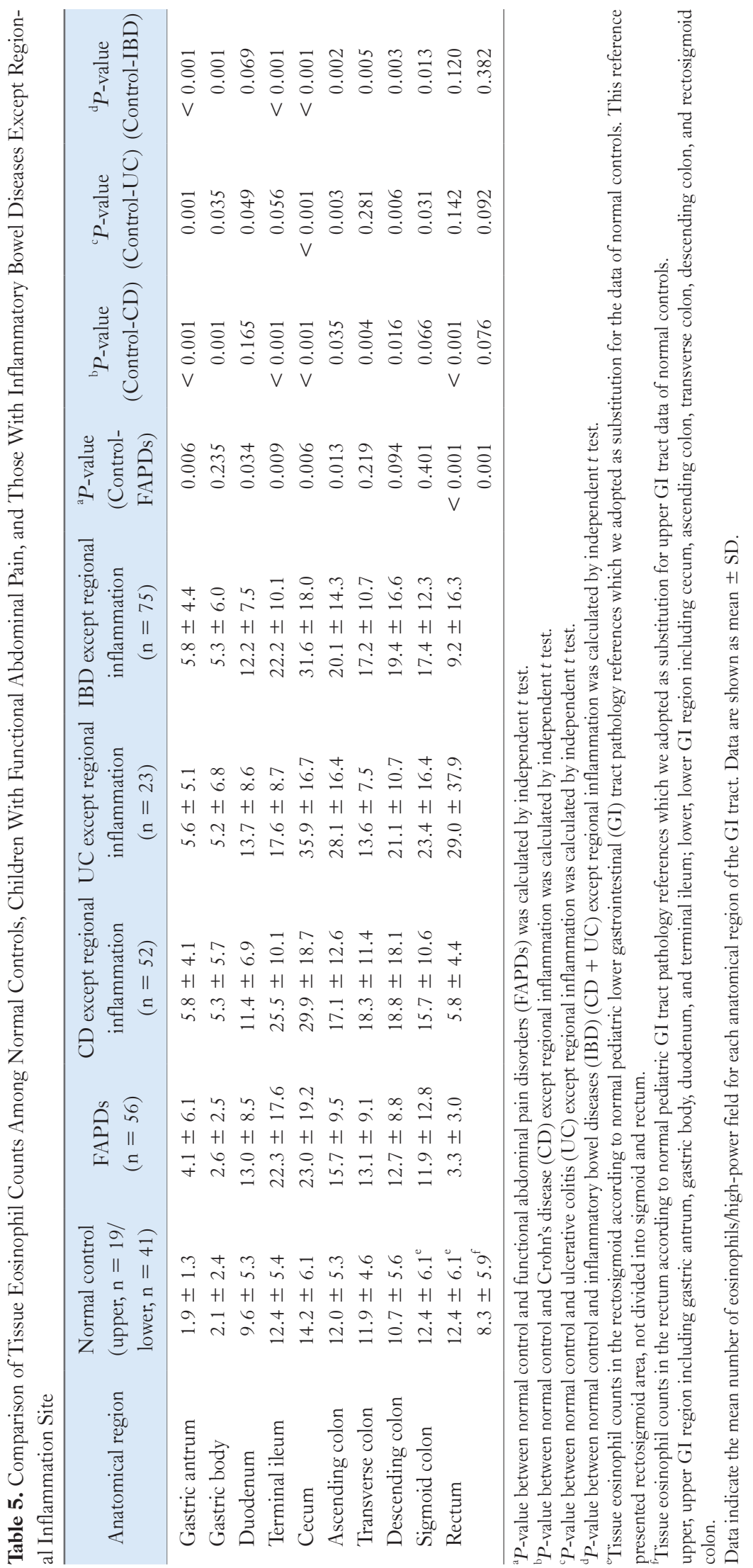


parison between CD pathology without macroscopic lesions and those studied as normal control showed that the eosinophil counts of the stomach (gastric antrum and body), terminal ileum, cecum, ascending colon, transverse colon, and descending colon were significantly higher in those with CD without macroscopic lesions than in those studied as normal controls (Table 5). A comparison between children with UC and normal controls after excluding the tissue specimens with macroscopically observed active inflamma- tory lesions showed that the eosinophil counts of the stomach (gastric antrum and body), duodenum, cecum, ascending colon, descending colon, and sigmoid colon were significantly higher in those with UC than those observed in normal controls (Table 5). Eosinophil counts of rectum in children with UC without macroscopic lesions were much higher than in those studied as normal controls, but this difference was not statistically significant because the number of eliminated slides was too large to show statistical power (Table 5).

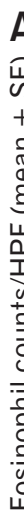

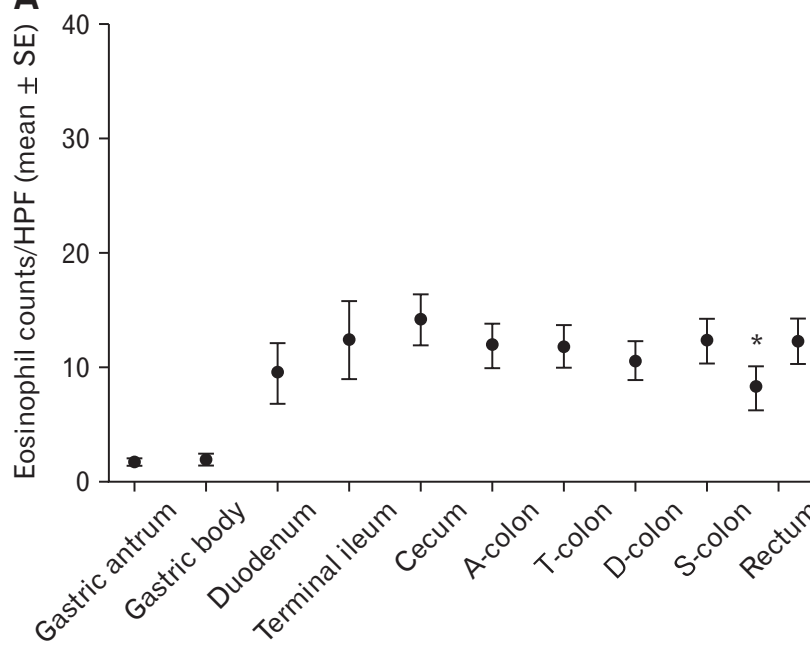

Cu

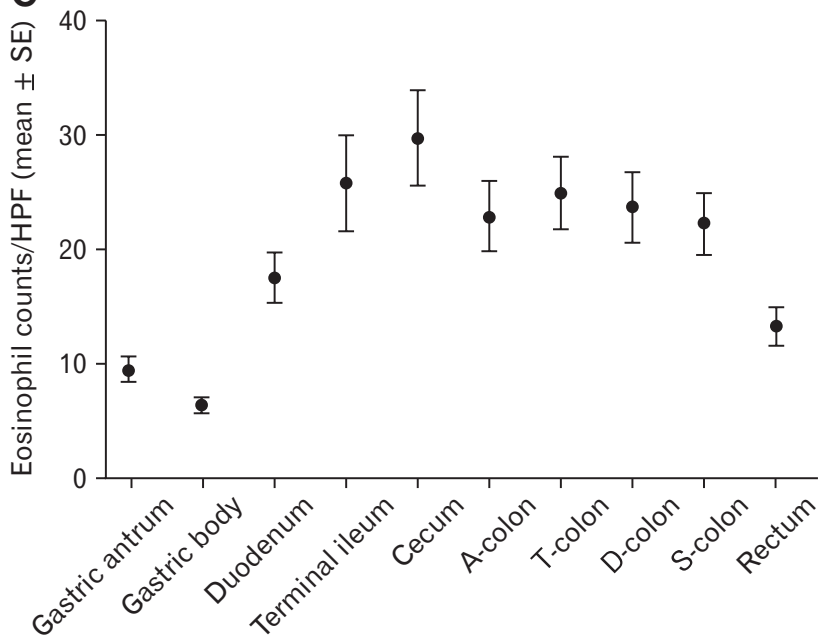

B

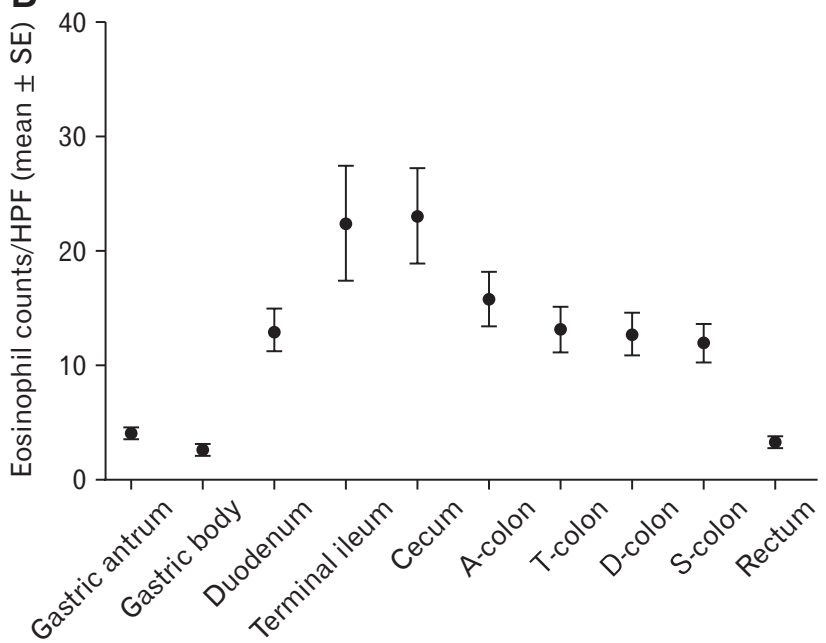

\section{D}

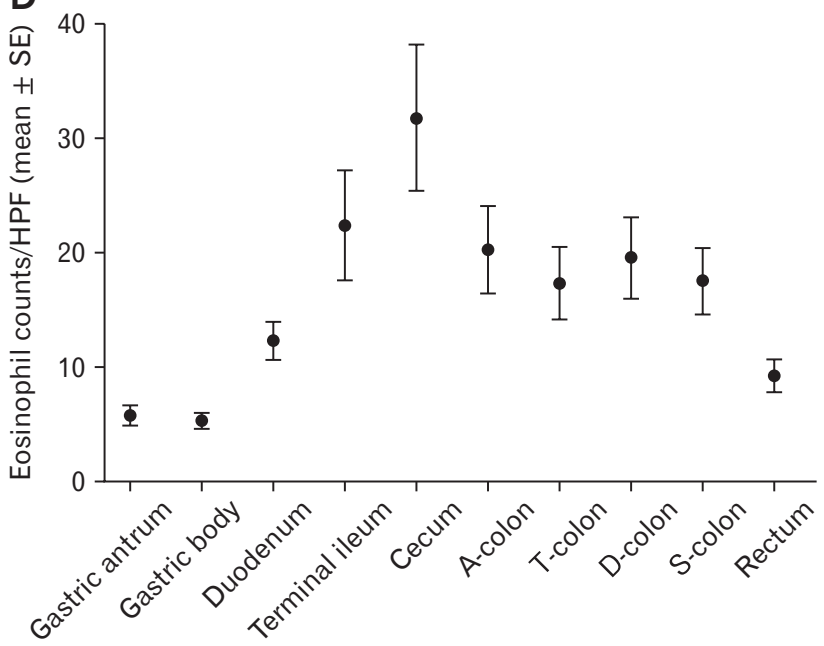

Figure 4. Eosinophils distribution of whole gastrointestinal (GI) tract in normal controls (A), functional abdominal pain disorders (FAPDs) (B), inflammatory bowel diseases (IBD) (C), and IBD after selecting the GI tract segments endoscopically did not show macroscopic IBD involvement (D). The graph shows the tendency that eosinophils increased from the stomach to the cecum and decreased from the cecum to the rectum along the colon. The degree of this tendency was more prominent in the FAPDs and IBD than that observed in normal controls. Eosinophil counts were significantly high in the order of IBD, FAPDs, and normal controls, regardless of endoscopically detected macroscopic IBD lesions. In the rectum, eosinophil counts according to both upper GI reference and lower GI reference were presented because lower GI references presented the "rectosigmoid area," with no clear division between the sigmoid and the rectum. HPF, high-power field; A-colon, ascending colon; T-colon, transverse colon; D-colon, descending colon; S-colon, sigmoid colon. The asterisk (*) means normal reference of rectum on the study by Debrosse et al. $^{12}$ 
Eosinophilic distributions of the entire GI tract in normal controls, children with FAPDs, those with IBD before and after eliminating GI segments with macroscopic IBD lesions on endoscopy are shown in Figure 4. Eosinophils are not uniformly distributed through the length of the intestine (Fig. 4). Eosinophils increased from the stomach to the cecum and again decreased from the cecum to the rectum along the colon and this tendency was more prominent in the FAPDs and IBD than that observed in normal controls (Fig. 4). Eosinophil counts were compared in each specific region of the GI tract and significantly high eosinophil counts of the GI tract were noted in the order of IBD, FAPDs, and normal controls, regardless of endoscopically detected macroscopic IBD lesions (Fig. $4)$.

\section{Comparison of Tissue Eosinophil Counts According to the Subtypes of Functional Abdominal Pain Disorders and Sex}

To compare tissue eosinophil counts in each specific region according to the subtypes of FAPDs, 56 children with FAPDs were classified into the 3 groups; IBS group $(n=37)$, overlap group $(\mathrm{IBS}+\mathrm{FD}$, IBS + FAP-NOS, IBS + AM; $\mathrm{n}=9)$, and nonIBS group (FAP-NOS, FD, and AM; n = 10) (Table 6). Eosinophil counts of each region of the GI tract were not significantly different among the 3 groups (Table 6).

To identify the difference in tissue eosinophil counts of GI tract between male and female patients, tissue eosinophil counts in each region were compared according to sex in 56 children with FAPDs.
Eosinophil counts of each region of the GI tract were not significantly different between boys and girls (Table 7).

\section{Discussion}

Eosinophils are known to be involved with immune response and affect tissue damage and repair processes. ${ }^{17}$ Because eosinophils contribute to multiple phases of the immune response, they can significantly influence disease processes. ${ }^{17}$ The accumulation and degradation of eosinophils causes neural stimulation and smooth muscle contraction, which consequently produces GI symptoms

Table 7. Comparison of Tissue Eosinophil Counts According to Sex of Children With Functional Abdominal Pain Disorders

\begin{tabular}{lccc}
\hline Anatomical region & Boys $(\mathrm{n}=39)$ & Girls $(\mathrm{n}=17)$ & $P$-value \\
\hline Gastric antrum & $4.0 \pm 6.7$ & $4.2 \pm 4.7$ & 0.502 \\
Gastric body & $2.5 \pm 2.6$ & $2.9 \pm 2.5$ & 0.444 \\
Duodenum & $13.5 \pm 12.0$ & $16.1 \pm 10.8$ & 0.273 \\
Terminal ileum & $26.1 \pm 19.3$ & $13.5 \pm 8.3$ & 0.136 \\
Cecum & $25.0 \pm 21.0$ & $16.1 \pm 8.5$ & 0.412 \\
Ascending colon & $16.4 \pm 10.6$ & $13.8 \pm 5.8$ & 0.776 \\
Transverse colon & $13.6 \pm 9.5$ & $11.7 \pm 8.5$ & 0.463 \\
Descending colon & $11.4 \pm 5.8$ & $15.7 \pm 13.3$ & 0.641 \\
Sigmoid colon & $9.9 \pm 5.7$ & $16.6 \pm 21.4$ & 0.224 \\
Rectum & $3.1 \pm 2.9$ & $3.6 \pm 3.2$ & 0.597 \\
\hline
\end{tabular}

${ }^{a} P$-value between normal control and functional abdominal pain disorders was calculated by Mann-Whitney $U$ test.

Table 6. Comparison of Tissue Eosinophil Counts Among the Subtypes of Functional Abdominal Pain Disorders

\begin{tabular}{|c|c|c|c|c|}
\hline Anatomical region & $\begin{array}{c}\text { IBS } \\
(\mathrm{n}=37)\end{array}$ & $\begin{array}{l}\text { Overlap } \\
(\mathrm{n}=9)\end{array}$ & $\begin{array}{l}\text { Non-IBS } \\
(\mathrm{n}=10)\end{array}$ & $P$-value ${ }^{a}$ \\
\hline Gastric antrum & $3.6 \pm 6.6$ & $5.4 \pm 6.4$ & $4.7 \pm 3.6$ & 0.249 \\
\hline Gastric body & $2.1 \pm 2.2$ & $3.9 \pm 3.8$ & $3.3 \pm 2.3$ & 0.204 \\
\hline Duodenum & $12.8 \pm 8.5$ & $21.4 \pm 22.8$ & $12.9 \pm 3.9$ & 0.716 \\
\hline Terminal ileum & $24.1 \pm 19.4$ & $23.0 \pm 8.0$ & $7.5 \pm 3.5$ & 0.242 \\
\hline Cecum & $22.5 \pm 20.7$ & $24.4 \pm 20.7$ & $24.0 \pm 13.3$ & 0.800 \\
\hline Ascending colon & $17.2 \pm 10.3$ & $13.0 \pm 6.4$ & $11.7 \pm 8.2$ & 0.518 \\
\hline Transverse colon & $14.0 \pm 10.3$ & $12.7 \pm 7.5$ & $9.3 \pm 2.4$ & 0.853 \\
\hline Descending colon & $13.3 \pm 8.5$ & $9.0 \pm 3.8$ & $14.2 \pm 12.9$ & 0.411 \\
\hline Sigmoid colon & $12.8 \pm 15.2$ & $10.1 \pm 7.5$ & $10.5 \pm 6.9$ & 0.916 \\
\hline Rectum & $3.3 \pm 3.1$ & $3.7 \pm 2.5$ & $2.8 \pm 3.2$ & 0.606 \\
\hline
\end{tabular}

${ }^{\mathrm{a}} \mathrm{P}$-value between normal control and with functional abdominal pain disorders (FAPDs) was calculated by Kruskal-Wallis test.

IBS, irritable bowel syndrome; FD, functional dyspepsia; FAP-NOS, functional abdominal pain not otherwise specified; AM, abdominal migraine.

Total of 56 children with FAPDs were classified as IBS $(\mathrm{n}=37), \operatorname{IBS}+$ FD $(\mathrm{n}=5), \operatorname{IBS}+$ FAP-NOS $(\mathrm{n}=3), \operatorname{IBS}+$ AM $(\mathrm{n}=1)$, FAP-NOS $(\mathrm{n}=6)$, FD $(\mathrm{n}$ $=3)$, and $\mathrm{AM}(\mathrm{n}=1)$.

Overlap group included IBS + FD $(\mathrm{n}=5)$, IBS + FAP-NOS $(\mathrm{n}=3)$, and IBS + AM $(\mathrm{n}=1)$.

Non-IBS group included FAP-NOS $(\mathrm{n}=6), \mathrm{FD}(\mathrm{n}=3)$, and AM $(\mathrm{n}=1)$. 
including abdominal pain in patients with FGIDs. ${ }^{10,18-20}$

To date, studies involving patients with IBS have not demonstrated a significant difference in the tissue eosinophil counts. ${ }^{6,21-23}$ Furthermore, histopathological studies in children with FAPDs are relatively rare, and pediatric studies have primarily been limited to studies investigating FD, focusing on the stomach and duodenum. ${ }^{8,92-27}$ Therefore, the histopathological features of the GI tract beyond the duodenum in children with FAPDs remain unclear. In the present study, we showed that eosinophil counts of the stomach (gastric antrum), small bowel (duodenum, terminal ileum), and proximal colon (cecum, ascending colon) were significantly higher in children with FAPDs including IBS than in normal controls. Our results suggest that GI eosinophils may be associated with FAPDs in children and may contribute to its pathogenesis.

Several studies have shown that mucosal eosinophilia was prominent in IBD patients compared to healthy controls or those with IBS. ${ }^{28}$ Furthermore, eosinophil counts or eosinophil cationic protein levels positively correlated with disease activity and negatively correlated with treatment response in IBD patients. ${ }^{17,29,30}$ Therefore, eosinophils are known to play a role in the pathogenesis of IBD, even though their exact relationship with IBD remains unclear. $^{11,28,31,32}$

We hypothesized that micro-inflammation may be the primary pathogenesis that causes GI symptoms in FAPDs. ${ }^{6,7,18,21,33-39}$ Based on this hypothesis, we presumed the density of inflammatory cells in the GI tract of the FAPDs group to be higher than that observed in normal controls without any inflammation and lower than that observed in the IBD group with definite inflammation of the GI tract. Study results showed significantly high eosinophil counts of the stomach and colon in the order of IBD, FAPDs, and normal controls, regardless of endoscopically detected macroscopic IBD lesions. Our study results are in agreement with those of previous studies reported by Bass et al, ${ }^{40}$ Pensabene et $\mathrm{al}^{41}$ and Flores et $\mathrm{al}^{42}$ in that eosinophil counts of the colonic area were significantly higher in children with IBD than in those with FAPDs or IBS or normal controls, although a study by Bass et $\mathrm{al}^{40}$ described that tissue eosinophil counts of the stomach were not significantly different between children with IBD and those with FAPDs, unlike our results. Furthermore, a study reported by Carvalho et $\mathrm{al}^{43}$ has shown the results consistent with those of our study in that high eosinophil counts of the colon showed a definite order in terms of the disease entity UC, followed by CD, non-inflamed CD, and IBS. Their results suggested that the macroscopic uninvolved mucosa may not be entirely normal in patients with IBD and that eosinophils could play some roles in the pathogenesis of the early lesions in IBD. ${ }^{43}$
To our knowledge, the present study is the first comparative study completed wherein the entire GI tract was evaluated to determine differences in tissue eosinophil infiltration in patients with FGIDs. A gradient of eosinophil density is known to exist from the proximal to the distal colon ${ }^{12,13,31,41,44}$; therefore, it is important that GI tract eosinophils should be analyzed through comparisons performed in each specific region, even in the colonic area. In several studies performed on adults with IBS, the biopsy specimens studied were primarily from the descending colon or rectosigmoid area. ${ }^{37}$ In several comparative studies involving patients with IBS and/ or IBD, some studies have not clearly described which part of the colon was analyzed, and others were performed with tissue obtained only from the rectosigmoid/rectal area. ${ }^{29,40-43}$ An inappropriate comparison of eosinophils within different sections of the colon can lead to inaccurate results. In this regard, our study involved assessment of the entire GI tract and compared eosinophils in each segment of the gut, which is strength of our study.

The present study has some limitations. First, this study could not recruit healthy individuals as normal controls. Although the comparison with age- and sex-matched healthy children as normal controls would be ideal, this was not performed because of ethical considerations regarding subjecting healthy children without any GI symptoms to endoscopic examination. Instead, we adopted normal reference values based on a previously published study, which evaluated endoscopic mucosal biopsies from each segment of the GI tract for children with no identified organic findings, and performed the final clinical assessment. Furthermore, we used two references ${ }^{12,13}$; one study as the upper GI tract reference and the other one as the lower GI tract reference. Since these reference groups did not comprise of Korean children, they may not accurately represent normal controls. The study reported by DeBrosse et $\mathrm{al}^{12}$ was already used as a normal reference in our previous study ${ }^{10}$ published in 2016 so it can be a good alternative as upper GI reference. However, we should find another normal reference to compare eosinophils of the colonic area because the study reported by DeBrosse et $\mathrm{al}^{12}$ does not provide information regarding the cecum and the descending and sigmoid colon. A study published in 2016 by Chernetsova et $\mathrm{al}^{45}$ showed eosinophil counts of the whole GI tract in healthy Canadian children, however the study used different stain methods, hematoxylin-phloxine-saffron and Giemsa stain to enhance cells detection, so their results present much higher cell counts than the study by DeBrosse et $\mathrm{al}^{12}$ and the study by Saad. ${ }^{13}$ The study by Chernestova et $\mathrm{al}^{45}$ compared their results with the aforementioned two studies and admitted differences. Thus, we used the study reported by DeBrosse et $\mathrm{al}^{12}$ as an upper GI refer- 
ence to compare eosinophil infiltration between the stomach and ileum, and another study reported by $\mathrm{Saad}^{13}$ as a lower GI reference to compare colonic eosinophil counts. Because of the study reported by Saad ${ }^{13}$ which we used as a lower GI pathology reference presented a 'rectosigmoid area,' and the region was not definitively divided into the sigmoid and rectum, it was difficult to accurately compare the eosinophil counts in the rectum. So, when the rectal eosinophils were compared using the reference values, rectal eosinophil counts reported in the study by DeBrosse et $\mathrm{al}^{12}$ were also presented as normal reference values in Table 4 and Table 5.

Second, this was a retrospective study with a relatively small sample size, and evaluated only eosinophils without other inflammatory cells. Third, the majority (46 [82.1\%]) of 56 children with FAPDs were patients with IBS. This is due to the fact that children with IBS mostly underwent colonoscopic examination. Further prospective studies including a larger number of children, balanced recruitment of other subtypes of FAPDs, and the analysis of additional cell types such as mast cells or intraepithelial lymphocytes are needed to confirm low-grade inflammation in children with FAPDs.

The present study evaluated tissue eosinophil infiltration throughout the entire GI tract in children with FAPDs compared to those with IBD, and to normal reference values in each specific region of the GI tract. Our results suggest GI tract eosinophilia may contribute to the development of childhood FAPDs as one of key mechanisms of the pathogenesis.

Acknowledgements: The authors would like to thank Dr Je Bong Lee at Seoul National University Bundang Hospital Medical Research Collaborating Center for her assistance in statistical analyses.

Financial support: This study was supported by a grant from Seoul National University Bundang Hospital Research Fund (Grant No. 02-2015-033).

\section{Conflicts of interest: None.}

Author contributions: Eun Hye Lee: conducting the study, collecting and interpreting data, drafting the manuscript; Hye Ran Yang: designing the study, interpreting data, drafting the manuscript; and Hye Seung Lee: conducting the study, collecting and interpreting data.

\section{References}

1. Korterink JJ, Diederen K, Benninga MA, Tabbers MM. Epidemiology of pediatric functional abdominal pain disorders: a meta-analysis. PLoS One 2015;10:e0126982.

2. Madani S, Parikh S, Madani RS, Krasaelap A. Long-term study of children with rome III functional gastrointestinal disorders managed symptomatically in a biopsychosocial model. Gastroenterology Res 2017;10:8491.

3. Horst S, Shelby G, Anderson J, et al. Predicting persistence of functional abdominal pain from childhood into young adulthood. Clin Gastroenterol Hepatol 2014;12:2026-2032.

4. Hyams JS, Di Lorenzo C, Saps M, Shulman RJ, Staiano A, van Tilburg M. Functional disorders: children and adolescents. Gastroenterology 2016;150:1456-1468, e2.

5. Schmulson MJ, Drossman DA. What is new in rome IV. J Neurogastroenterol Motil 2017;23:151-163.

6. Ford AC, Talley NJ. Mucosal inflammation as a potential etiological factor in irritable bowel syndrome: a systematic review. J Gastroenterol 2011;46:421-431.

7. Vanheel H, Vicario M, Vanuytsel T, et al. Impaired duodenal mucosal integrity and low-grade inflammation in functional dyspepsia. Gut 2014;63:262-271.

8. Wauters L, Nightingale S, Talley NJ, Sulaiman B, Walker MM. Functional dyspepsia is associated with duodenal eosinophilia in an Australian paediatric cohort. Aliment Pharmacol Ther 2017;45:1358-1364.

9. Schurman JV, Singh M, Singh V, Neilan N, Friesen CA. Symptoms and subtypes in pediatric functional dyspepsia: relation to mucosal inflammation and psychological functioning. J Pediatr Gastroenterol Nutr 2010;51:298-303.

10. Lee EH, Yang HR, Lee HS. Analysis of gastric and duodenal eosinophils in children with abdominal pain related functional gastrointestinal disorders according to rome III criteria. J Neurogastroenterol Motil 2016;22:459-469.

11. Yantiss RK. Eosinophils in the GI tract: how many is too many and what do they mean? Mod Pathol 2015;28(suppl 1):S7-S21.

12. DeBrosse CW, Case JW, Putnam PE, Collins MH, Rothenberg ME. Quantity and distribution of eosinophils in the gastrointestinal tract of children. Pediatr Dev Pathol 2006;9:210-218.

13. Saad AG. Normal quantity and distribution of mast cells and eosinophils in the pediatric colon. Pediatr Dev Pathol 2011;14:294-300.

14. Daperno M, D'Haens G, Van Assche G, et al. Development and validation of a new, simplified endoscopic activity score for Crohn's disease: the SES-CD. Gastrointest Endosc 2004;60:505-512.

15. Travis SP, Schnell D, Krzeski P, et al. Developing an instrument to assess the endoscopic severity of ulcerative colitis: the Ulcerative Colitis Endoscopic Index of Severity (UCEIS). Gut 2012;61:535-542.

16. Travis SP, Schnell D, Krzeski P, et al. Reliability and initial validation of the ulcerative colitis endoscopic index of severity. Gastroenterology 2013;145:987-995.

17. Travers J, Rothenberg ME. Eosinophils in mucosal immune responses. Mucosal Immunol 2015;8:464-475.

18. Wang X, Li X, Ge W, et al. Quantitative evaluation of duodenal eo- 
sinophils and mast cells in adult patients with functional dyspepsia. Ann Diagn Pathol 2015;19:50-56.

19. Lee KJ, Tack J. Duodenal implications in the pathophysiology of functional dyspepsia. J Neurogastroenterol Motil 2010;16:251-257.

20. Powell N, Walker MM, Talley NJ. Gastrointestinal eosinophils in health, disease and functional disorders. Nat Rev Gastroenterol Hepatol 2010;7:146-156

21. Walker MM, Talley NJ, Prabhakar M, et al. Duodenal mastocytosis, eosinophilia and intraepithelial lymphocytosis as possible disease markers in the irritable bowel syndrome and functional dyspepsia. Aliment Pharmacol Ther 2009;29:765-773.

22. Piche T, Saint-Paul MC, Dainese R, et al. Mast cells and cellularity of the colonic mucosa correlated with fatigue and depression in irritable bowel syndrome. Gut 2008;57:468-473.

23. O'Sullivan M, Clayton N, Breslin NP, et al. Increased mast cells in the irritable bowel syndrome. Neurogastroenterol Motil 2000;12:449-457.

24. Friesen CA, Andre L, Garola R, Hodge C, Roberts C. Activated duodenal mucosal eosinophils in children with dyspepsia: a pilot transmission electron microscopic study. J Pediatr Gastroenterol Nutr 2002;35:329333.

25. Friesen CA, Neilan NA, Schurman JV, Taylor DL, Kearns GL, AbdelRahman SM. Montelukast in the treatment of duodenal eosinophilia in children with dyspepsia: effect on eosinophil density and activation in relation to pharmacokinetics. BMC Gastroenterol 2009;9:32.

26. Friesen CA, Lin Z, Singh M, et al. Antral inflammatory cells, gastric emptying, and electrogastrography in pediatric functional dyspepsia. Dig Dis Sci 2008;53:2634-2640.

27. Friesen CA, Kearns GL, Andre L, Neustrom M, Roberts CC, AbdelRahman SM. Clinical efficacy and pharmacokinetics of montelukast in dyspeptic children with duodenal eosinophilia. J Pediatr Gastroenterol Nutr 2004;38:343-351.

28. Mehta P, Furuta GT. Eosinophils in gastrointestinal disorders: eosinophilic gastrointestinal diseases, celiac disease, inflammatory bowel diseases, and parasitic infections. Immunol Allergy Clin North Am 2015;35:413437.

29. Zezos P, Patsiaoura K, Nakos A, et al. Severe eosinophilic infiltration in colonic biopsies predicts patients with ulcerative colitis not responding to medical therapy. Colorectal Dis 2014;16:O420-O430.

30. Kristjánsson G, Venge P, Wanders A, Lööf L, Hällgren R. Clinical and subclinical intestinal inflammation assessed by the mucosal patch technique: studies of mucosal neutrophil and eosinophil activation in inflammatory bowel diseases and irritable bowel syndrome. Gut 2004;53:18061812.
31. Woodruff SA, Masterson JC, Fillon S, Robinson ZD, Furuta GT. Role of eosinophils in inflammatory bowel and gastrointestinal diseases. J Pediatr Gastroenterol Nutr 2011;52:650-661.

32. Katsanos KH, Zinovieva E, Lambri E, Tsianos EV. Eosinophilic-Crohn overlap colitis and review of the literature. J Crohns Colitis 2011;5:256261.

33. Keely S, Walker MM, Marks E, Talley NJ. Immune dysregulation in the functional gastrointestinal disorders. Eur J Clin Invest 2015;45:13501359.

34. Talley NJ, Walker MM, Holtmann G. Functional dyspepsia. Curr Opin Gastroenterol 2016;32:467-473.

35. Akiho H, Ihara E, Nakamura K. Low-grade inflammation plays a pivotal role in gastrointestinal dysfunction in irritable bowel syndrome. World J Gastrointest Pathophysiol 2010;1:97-105.

36. Walker MM, Warwick A, Ung C, Talley NJ. The role of eosinophils and mast cells in intestinal functional disease. Curr Gastroenterol Rep 2011;13:323-330.

37. Bashashati M, Moossavi S, Cremon C, et al. Colonic immune cells in irritable bowel syndrome: a systematic review and meta-analysis. Neurogastroenterol Motil 2018;30.

38. Wouters MM, Vicario M, Santos J. The role of mast cells in functional GI disorders. Gut 2016;65:155-168.

39. Ohman L, Simrén M. Pathogenesis of IBS: role of inflammation, immunity and neuroimmune interactions. Nat Rev Gastroenterol Hepatol 2010;7:163-173.

40. Bass JA, Friesen CA, Deacy AD, et al. Investigation of potential early histologic markers of pediatric inflammatory bowel disease. BMC Gastroenterol 2015;15:129.

41. Pensabene L, Brundler MA, Bank JM, Di Lorenzo C. Evaluation of mucosal eosinophils in the pediatric colon. Dig Dis Sci 2005;50:221-229.

42. Flores C, Francesconi CF, Meurer L. Quantitative assessment of $\mathrm{CD} 30+$ lymphocytes and eosinophils for the histopathological differential diagnosis of inflammatory bowel disease. J Crohns Colitis 2015;9:763768.

43. Carvalho AT, Elia CC, de Souza HS, et al. Immunohistochemical study of intestinal eosinophils in inflammatory bowel disease. J Clin Gastroenterol 2003;36:120-125.

44. Hurrell JM, Genta RM, Melton SD. Histopathologic diagnosis of eosinophilic conditions in the gastrointestinal tract. Adv Anat Pathol 2011;18:335-348.

45. Chernetsova E, Sullivan K, de Nanassy J, et al. Histologic analysis of eosinophils and mast cells of the gastrointestinal tract in healthy Canadian children. Hum Pathol 2016;54:55-63. 